\title{
Decreasing Hospital Observation Time for Febrile Infants
}

\author{
Sanyukta Desai, MD, MSc*, Tara Calhoun, MD2,3, Tina Sosa, MD², Joshua D Courter, PharmD4, Alexandra Letsinger, RN5 , \\ Matthew Le, MD', Amy Schubert, RN5, Lindsay Zaremba, MD7, Samir S Shah, MD, MSCE², Karen Jerardi, MD, MEd², \\ Angela M Statile, MD, MEd ${ }^{2,3}$, Ndidi I Unaka, MD, MEd ${ }^{2,3}$
}

\begin{abstract}
${ }^{1}$ Division of Hospital Medicine, Department of Pediatrics, Seattle Children's Hospital, University of Washington School of Medicine, Seattle, Washington; ${ }^{2}$ Division of Hospital Medicine, Cincinnati Children's Hospital Medical Center, Cincinnati, Ohio; ${ }^{3}$ Department of Pediatrics, University of Cincinnati College of Medicine, Cincinnati, Ohio; ${ }^{4}$ Division of Pharmacy, Cincinnati Children's Hospital Medical Center, Cincinnati, Ohio; ${ }^{5}$ Cincinnati Children's Hospital Medical Center, Cincinnati, Ohio; ${ }^{6}$ Section of Hospital Medicine, Department of Pediatrics, University of Oklahoma Health Science Center, Oklahoma City, Oklahoma; ' Division of Hospital Medicine, Department of Pediatrics, University Hospital Rainbow Babies and Children's Hospital, Cleveland Ohio; ${ }^{8}$ Division of Infectious Diseases, Cincinnati Children's Hospital Medical Center, Cincinnati, Ohio.
\end{abstract}

BACKGROUND: Febrile infants aged 0 to 60 days are often hospitalized for a 36-to-48 hour observation period to rule out invasive bacterial infections (IBI). Evidence suggests that monitoring blood and cerebrospinal fluid (CSF) cultures for 24 hours may be appropriate for most infants. We aimed to decrease the average culture observation time (COT) from 38 to 30 hours among hospitalized infants 0 to 60 days old over 12 months.

METHODS: This quality improvement initiative occurred at a large children's hospital, in conjunction with development of a multidisciplinary evidence-based guideline for the management of febrile infants. We included infants aged 0 to 60 days admitted with fever without a clear infectious source. We excluded infants who had positive blood, urine, or CSF cultures within 24 hours of incubation and infants who were hospitalized for other indications (eg, bronchiolitis). Interventions included guideline dissemination, education regarding laboratory monitoring practices, standardized order sets, and near-time identification of failures. Our primary outcome was COT, defined as time between initiation of culture incubation and hospital discharge in hours. Interventions were tracked on an annotated statistical process control chart. Our balancing measure was identification of IBI after hospital discharge.

RESULTS: In our cohort of 184 infants aged 0 to 60 days, average COT decreased from 38 hours to 32 hours after structured guideline dissemination and order-set standardization; this decrease was sustained over 17 months. $\mathrm{IBI}$ was not identified in any patients after discharge.

CONCLUSIONS: Implementation of an evidence-based guideline through education, transparency of laboratory procedures, creation of standardized order sets, and neartime feedback was associated with shorter COT for febrile infants aged 0 to 60 days. Journal of Hospital Medicine 2021;16:267-273. ( 2021 Society of Hospital Medicine

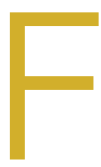

ebrile infants aged 0 to 60 days often undergo diagnostic testing to evaluate for invasive bacterial infections (IBI; ie, bacteremia and meningitis) and are subsequently hospitalized pending culture results. Only $1 \%$ to $2 \%$ of infants 0 to 60 days old have an $\mid \mathrm{BI},^{1-3}$ and most hospitalized infants are discharged once physicians feel confident that pathogens are unlikely to be isolated from blood and cerebrospinal fluid (CSF) cultures. Practice regarding duration of hospitalization while awaiting blood and CSF culture results is not standardized in this population. Longer hospitalizations can lead to increased costs and familial stress, including difficulty with breastfeeding and anxiety in newly postpartum mothers. ${ }^{4,5}$

*Corresponding Author: Sanyukta Desai, MD; Email: sanyukta.desai@seattlechildrens.org; Telephone: 206-987-7370.

Published online first April 20, 2021.

Find additional supporting information in the online version of this article.

Received: October 13, 2020; Revised: January 4, 2021; Accepted: January 18, 2021

๑ 2021 Society of Hospital Medicine DOI 10.12788/jhm.3593
In 2010, an institutional evidence-based guideline for the management of febrile infants aged 0 to 60 days recommended discharge after 36 hours of observation if all cultures were negative. ${ }^{6}$ However, recent studies demonstrate that $85 \%$ to 93\% of pathogens in blood and CSF cultures grow within 24 hours of incubation..$^{7-9}$ Assuming a $2 \%$ prevalence of $|B|$, if $15 \%$ of pathogens were identified after 24 hours of incubation, only one out of 333 infants would have an IBI identified after 24 hours of hospital observation.?

Furthermore, a review of our institution's electronic health records (EHR) over the past 5 years revealed that an observation period of 24 hours would have resulted in the discharge of three infants with an IBI. Two infants had bacteremia; both were discharged from the emergency department (ED) without antibiotics, returned to care after cultures were reported positive at 27 hours, and had no adverse outcomes. The third infant had meningitis, but also had an abnormal CSF Gram stain, which led to a longer hospitalization.

In 2019, our institution appraised the emerging literature and institutional data supporting the low absolute risk of missed $\mid \mathrm{BI}$, and also leveraged local consensus among key stakehold- 
ers to update its evidence-based guideline for the evaluation and management of febrile infants aged 60 days and younger. The updated guideline recommends that clinicians consider discharging well-appearing neonates and infants if blood and CSF cultures remain negative at 24 hours. ${ }^{10}$ The objective of this study was to decrease the average hospital culture observation time (COT; culture incubation to hospital discharge) from 38 to 30 hours over a 12-month period in febrile infants aged 0 to 60 days.

\section{METHODS}

\section{Context}

Improvement efforts were conducted at Cincinnati Children's Hospital Medical Center (CCHMC), a large, urban, academic hospital that admitted more than 8,000 noncritically ill patients to the hospital medicine (HM) service from July 1, 2018, through June 30, 2019. Hospital medicine teams, located at both the main and satellite campuses, are staffed by attending physicians, fellows, residents, medical students, and nurse practitioners. The two campuses, which are about 20 miles apart, share clinician providers but have distinct nursing pools.

Microbiology services for all CCHMC patients are provided at the main campus. Blood and CSF cultures at the satellite campus are transported to the main campus for incubation and monitoring via an urgent courier service. The microbiology laboratory at CCHMC uses a continuous monitoring system for blood cultures (BACT/ALERT Virtuo, BioMérieux). The system automatically alerts laboratory technicians of positive cultures; these results are reported to clinical providers within $30 \mathrm{~min}$ utes of detection. Laboratory technicians manually evaluate CSF cultures once daily for 5 days.

\section{Improvement Team}

Our improvement team included three HM attending physicians; two HM fellows; a pediatric chief resident; two nurses, who represented nursing pools at the main and satellite campuses; and a clinical pharmacist, who is a co-leader of the antimicrobial stewardship program at CCHMC. Supporting members for the improvement team included the CCHMC laboratory director; the microbiology laboratory director; an infectious disease physician, who is a co-leader of the antimicrobial stewardship program; and nursing directors of the HM units at both campuses.

\section{Evidence-Based Guideline}

Our improvement initiative was based on recommendations from the updated CCHMC Evidence-Based Care Guideline for Management of Infants 0 to 60 days with Fever of Unknown Source. ${ }^{10}$ This guideline, published in May 2019, was developed by a multidisciplinary working group composed of key stakeholders from HM, community pediatrics, emergency medicine, the pediatric residency program, infectious disease, and laboratory medicine. Several improvement team members were participants on the committee that published the evidence-based guideline. The committee first performed a systematic literature review and critical appraisal of the literature. Care recommendations were formulated via a consensus process directed by best evidence, patient and family preferences, and clinical expertise; the recommendations were subsequently reviewed and approved by clinical experts who were not involved in the development process.

Based on evidence review and multistakeholder consensus, the updated guideline recommends clinicians consider discharging neonates and infants aged 60 days and younger if there is no culture growth after an observation period of 24 hours (as documented in the EHR) and patients are otherwise medically ready for discharge (ie, well appearing with adequate oral intake). ${ }^{10,11}$ In addition, prior to discharge, there must be a documented working phone number on file for the patient's parents/guardians, an established outpatient follow-up plan within 24 hours, and communication with the primary pediatrician who is in agreement with discharge at 24 hours.

\section{Study Population}

Infants 0 to 60 days old who had a documented or reported fever without an apparent source based on history and physical exam upon presentation to the ED, and who were subsequently admitted to the HM service at CCHMC between October 30, 2018, and July 10, 2020, were eligible for inclusion. We excluded infants who were admitted to other clinical services (eg, intensive care unit); had organisms identified on blood, urine, or CSF culture within 24 hours of incubation; had positive herpes simplex virus testing; had skin/soft tissue infections or another clearly documented source of bacterial infection; or had an alternative indication for hospitalization (eg, need for intravenous fluid or deep suctioning) after cultures had incubated for 24 hours. Infants who had a positive blood, urine, or CSF culture result after 24 hours of incubation were included in the study population. Organisms were classified as pathogen or contaminant based on treatment decisions made by the care team.

\section{Improvement Activities}

Key drivers critical to success of the improvement efforts were: (1) clearly defined standard of care for duration of observation in febrile infants 0 to 60 days old; (2) improved understanding of microbiology lab procedures; (3) effective communication of discharge criteria between providers and nurses; and (4) transparency of data with feedback (Figure 1). The corresponding interventions were executed using Plan-Do-Study-Act (PDSA) cycles as follows:

\section{Education and Structured Dissemination of Evidence-Based Guideline}

The CCHMC febrile infant guideline ${ }^{10}$ was disseminated to HM physicians, residents, and nurses via the following means: (1) in-person announcements at staff meetings and educational conferences, (2) published highlights from the guideline in weekly newsletters, and (3) email announcements. Additionally, members of the study team educated HM attending physicians, nursing staff from the medical units at both campuses, and resident physicians about recent studies demonstrating safety of shorter length of stay (LOS) in febrile infants aged 


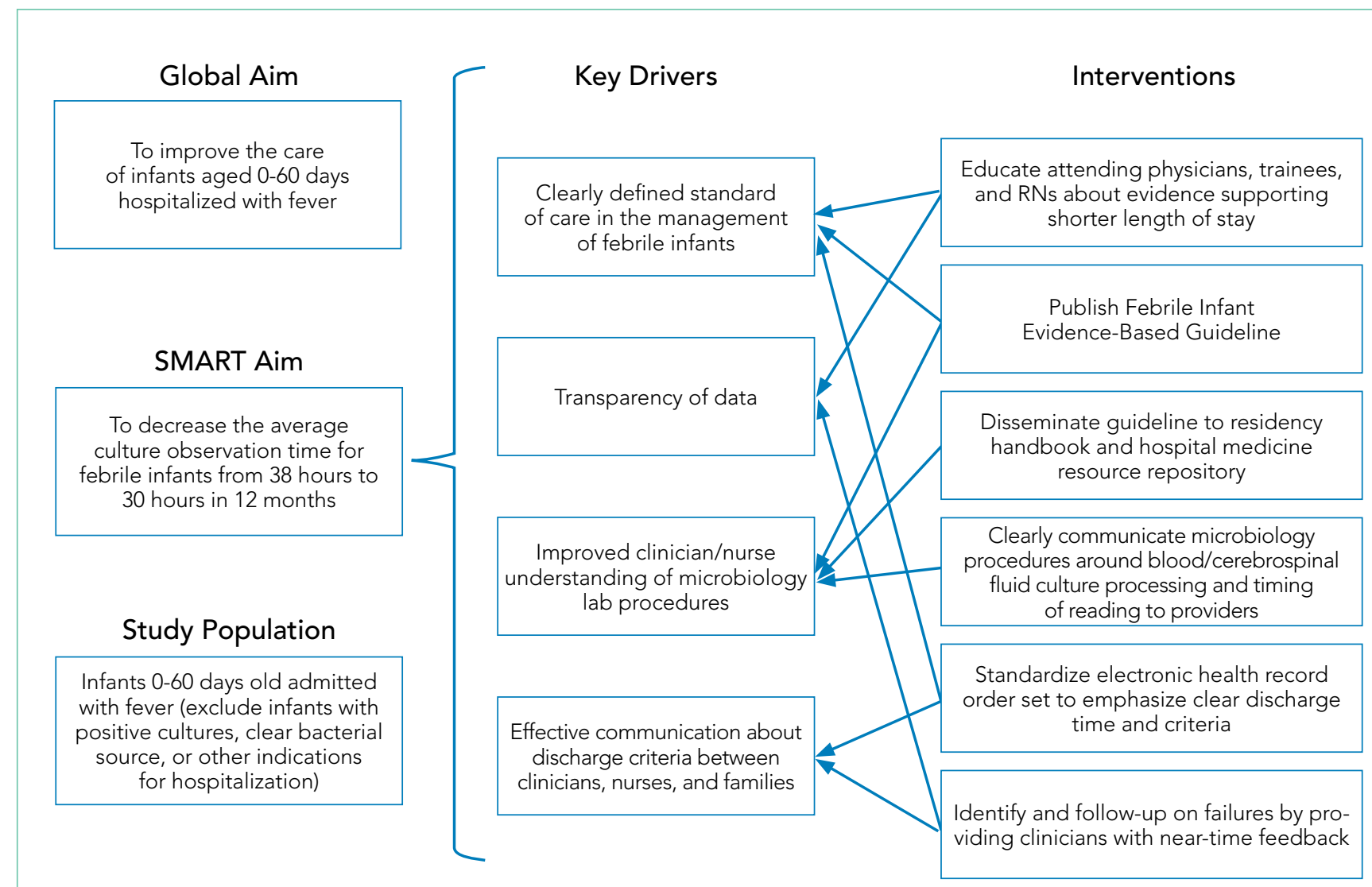

FIG 1. Key Driver Diagram Detailing Essential Drivers and Interventions Aimed at Reducing Culture Observation Time in Infants Aged 60 Days and Younger Hospitalized With Fever

0 to 60 days. The study team also provided residents, physicians, and nurses with data on the number of positive blood and CSF cultures and outcomes of patients at CCHMC within the past 5 years. In addition, team members led a journal club for residents discussing an article ${ }^{7}$ describing time-to-positivity of blood and CSF cultures in febrile infants. For ongoing engagement, the evidence-based guideline and a detailed explanation of microbiology procedures were published in the resident handbook, an internal resource that includes vital clinical pearls and practice guidelines across specialties. (Each resident receives an updated hard copy each year, and there is also an online link to the resource in the EHR.) Information about the guideline and COT was also included in the monthly chief resident's orientation script, which is relayed to all residents on the first day of their HM rotation.

\section{Clear Communication of Microbiology Procedures}

Team members created a detailed process map describing the processing protocols for blood and CSF cultures collected at both CCHMC campuses. This information was shared with HM attending physicians and nurses via in-person announcements at staff meetings, flyers in team workrooms, and email communications. Residents received information on microbiology protocols via in-person announcements at educational confer- ences and dissemination in the weekly residency newsletter. Important information communicated included:

1. Definition of culture start time. We conveyed that there may be a delay of up to 4 hours between culture collection at the satellite campus and culture incubation at the main campus laboratory. As a result, the time of blood or CSF sample arrival to the main campus laboratory was a more accurate reflection of the culture incubation start time than the culture collection time.

2. Explanation of CSF culture processing. We discussed the process by which these cultures are plated upon arrival at the microbiology laboratory and read once per day in the morning. Therefore, a culture incubated at midnight would be evaluated once at 9 hours and not again until 33 hours.

\section{Modification of Febrile Infant Order Set}

Enhancements to the febrile infant order set improved communication and cultivated a shared mental model regarding discharge goals among all members of the care team. The EHR order set for febrile infants was updated as follows: (1) mandatory free-text fields that established the culture start time for blood and CSF cultures were added, (2) culture start time was clearly defined (ie, the time culture arrives at the main campus laboratory), and (3) a change was made in the default 
discharge criteria" to "culture observation for 24 hours," with the ability to modify COT (Appendix Figure 1). We embedded hyperlinks to the guideline and microbiology process map within the updated order set, which allowed providers to easily access this information and refresh their knowledge of the recommendations (Appendix Figure 1).

\section{Identification of Failures and Follow-up With Near-Time Feedback}

All cases of febrile infants were tracked weekly. For infants hospitalized longer than 24 hours, the study team contacted the discharging clinicians to discuss reasons for prolonged hospitalization, with an emphasis on identifying system-level barriers to earlier discharge.

\section{Study of the Interventions}

The institutional microbiology database was queried weekly to identify all infants 0 to 60 days old who had a blood culture obtained and were hospitalized on the HM service. Study team members conducted targeted EHR review to determine whether patients met exclusion criteria and to identify reasons for prolonged COT. Baseline data were collected retrospectively for a 3-month period prior to initiation of improvement activities. During the study period, queries were conducted weekly and reviewed by study team members to evaluate the impact of improvement activities and to inform new interventions.

\section{Measures}

Our primary outcome measure was COT, defined as the hours between final culture incubation and hospital discharge. The operational definition for "final culture incubation" was the documented time of arrival of the last collected culture to the microbiology laboratory. Our goal COT was 30 hours to account for a subset of patients whose blood and/or CSF culture were obtained overnight (ie, after 9 PM), since subsequent discharge times would likely and practically be delayed beyond 24 hours. Our secondary outcome measure was LOS, defined as the time between ED arrival and hospital discharge. Process measures included the proportion of patients for whom the febrile infant EHR order set was used and the proportion of patients for whom medical discharge criteria (ie, blood and CSF culture observed for " $x x^{\prime \prime}$ hours) and culture incubation start times were entered using the order set. Balancing measures included identification of IBI after hospital discharge, 48-hour ED revisits, and 7-day hospital readmissions.

\section{Analysis}

Measures were evaluated using statistical process control charts and run charts, and Western Electric rules were employed to determine special cause variation. ${ }^{12}$ Annotated X-bar $\mathrm{S}$ control charts tracked the impact of improvement activities on average COT and LOS for all infants. Given that a relatively small number of patients (ie, two to four) met inclusion criteria each week, average COT was calculated per five patients.

This study was considered exempt from review by the CCHMC Institutional Review Board.

\section{RESULTS}

Of the 184 infants in this study, 46 were included as part of baseline data collection, and 138 were included during the intervention period. The median age was 26.6 days (range, 3-59 days); $52 \%$ of patients were female; two-thirds were non-Hispanic White; $22 \%$ were Black, and 5\% were Hispanic (Appendix Table).

Average COT decreased from 38 hours to 32 hours with improvement activities (Figure 2) and was sustained for a total of 17 months. There were small decreases in COT after initial education was provided to attendings, nurses, and residents. However, the greatest sustained decreases in COT occurred after dissemination of the published evidence-based guideline and standardization of the EHR order set. Average LOS decreased from 42 hours to 36 hours (Figure 3). Among the total cohort, $34 \%$ of infants were admitted to the satellite campus. At the satellite and main campuses, median COT was 28 hours and 35 hours, respectively (Appendix Figure 2).

After the launch of the updated order set, median usage of the EHR order set increased from $50 \%$ to $80 \%$. Medical discharge criteria were entered for 80 (96\%) of the 83 patients for whom the updated order set was applied; culture incubation start times were entered for 78 (94\%) of these patients.

No infants in our cohort were found to have IBI after hospital discharge. There were no ED revisits within 48 hours of discharge, and there were no hospital readmissions within 7 days of index discharge. Furthermore, none of the patients included in the study had growth of a pathogenic organism after 24 hours.

Of the 138 infants hospitalized during the intervention period, 77 (56\%) had a COT greater than 30 hours. Among these 77 patients, $49(64 \%)$ had their final culture incubated between 9 PM and 4 AM; Furthermore, 11 (14\%) had missing, abnormal, pretreated, or uninterpretable CSF studies, 7 (9\%) had ongoing fevers, and 4 (5\%) remained hospitalized due to family preference or inability to obtain timely outpatient follow-up.

\section{DISCUSSION}

Our study aimed to decrease the average COT from 38 hours to 30 hours among hospitalized infants aged 60 days and younger over a period of 12 months. An intervention featuring implementation of an evidence-based guideline through education, laboratory procedure transparency, creation of a standardized EHR order set, and near-time feedback was associated with a shorter average COT of 32 hours, sustained over a 17-month period. No infants with bacteremia or meningitis were inappropriately discharged during this study.

\section{Interpretation}

Prior to our improvement efforts, most febrile infants at CCHMC were observed for at least 36 hours based on a prior institutional guideline, ${ }^{6}$ despite recent evidence suggesting that most pathogens in blood and CSF cultures grow within 24 hours of incubation. ${ }^{7-9}$ The goal of this improvement initiative was to bridge the gap between emerging evidence and clinical practice by developing and dissemi- 


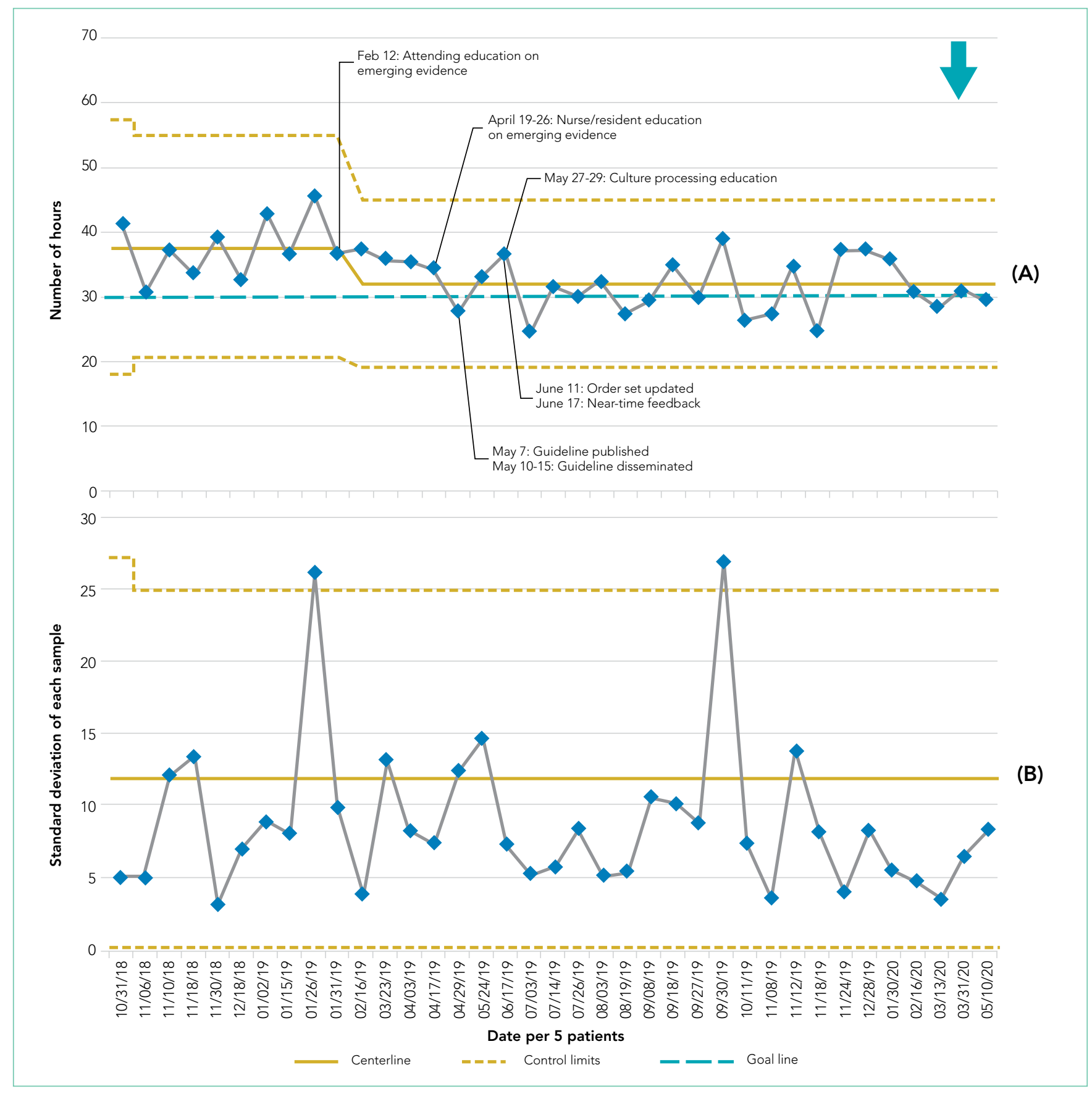

FIG 2. X-Bar S Control Chart Displaying Average Culture Observation Time per Five Admitted Febrile Infants Aged 60 Days and Younger. Each plotted point represents the average culture observation time (COT) per five infants, and the date listed represents earliest date of admission within the group. Panel A demonstrates changes in COT (in hours) over time, with interventions annotated and the centerline representing average COT. Panel B represents the SD in COT of each group, with the centerline representing average SD for the study population.

nating an updated evidence-based guideline to safely decrease the hospital observation time in febrile infants aged 60 days and younger.

Similar to previous studies aimed at improving diagnosis and management among febrile infants, ${ }^{13-16}$ generation and structured dissemination of an institutional evidence-based guideline was crucial to safely shortening COT in our population. These prior studies established a goal COT of 36 to
42 hours for hospitalized febrile infants. ${ }^{13,15,16}$ Our study incorporated emerging evidence and local experience into an updated evidence-based practice guideline to further reduce COT to 32 hours for hospitalized infants. Key factors contributing to our success included multidisciplinary engagement, specifically partnering with nurses and resident physicians in designing and implementing our initiatives. Furthermore, improved transparency of culture monitoring practices allowed 


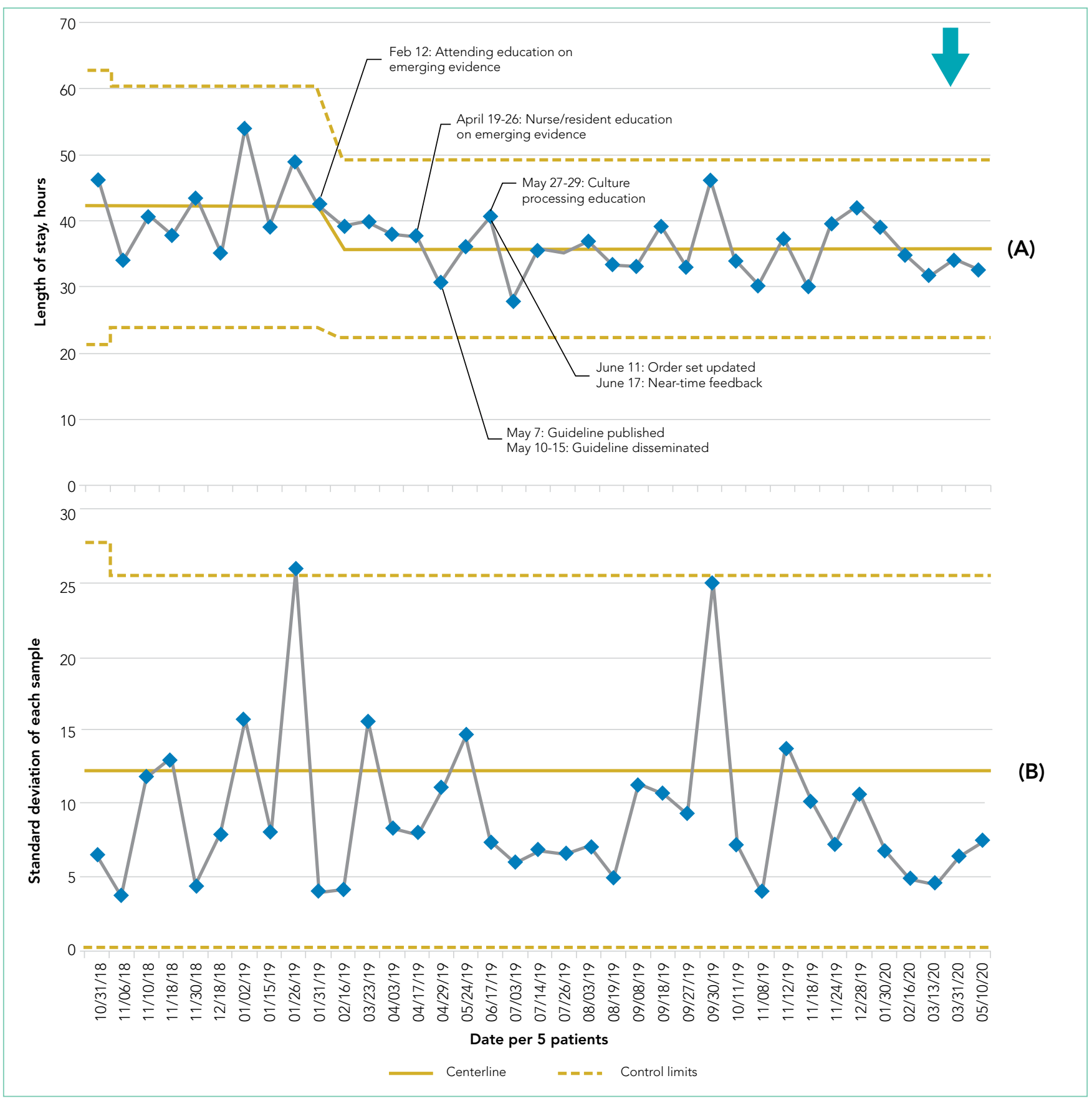

FIG 3. X-Bar S Control Chart Displaying Average Length of Stay From Emergency Department Arrival to Hospital Discharge per Five Admitted Febrile Infants Aged 60 Days and Younger. Each plotted point represents the average length of stay (LOS) per five infants; the date listed represents earliest date of admission within the group. Panel A demonstrates changes in LOS (in hours) over time, with interventions annotated and the centerline representing average LOS. Panel B represents the SD in LOS of each group, with the centerline representing average SD for the study population.

clinicians to better understand the recommended observation periods. Finally, we employed a standardized EHR order set as a no-cost, one-time, high-reliability intervention to establish 24 hours of culture monitoring as the default and to enhance transparency around start time for culture incubation.

Average COT remained stable at 32 hours for 17 months after initiation of the intervention. During the intervention period, $64 \%$ patients with hospital stays longer than 30 hours had cultures obtained between 9 PM to 4 AM. These patients often remained hospitalized for longer than 30 hours to allow for a daytime hospital discharge. Additionally, CSF cultures were only monitored manually once per day between $8 \mathrm{AM}$ and $10 \mathrm{AM}$. As a result, CSF cultures obtained in the evening (eg, 9 PM) would be evaluated once at roughly 12 hours of incubation, and then the following morning at 36 hours of incubation. In cases where CSF studies (eg, cell count, protein, Gram stain) were abnormal, uninterpre- 
table, or could not be obtained, clinicians monitored CSF cultures closer to 36 hours from incubation. While evidence-based guidelines and local data support safe early discharge of febrile infants, clinicians presented with incomplete or uninterpretable data were appropriately more likely to observe infants for longer periods to confirm negative cultures.

\section{Limitations}

The study has several limitations. First, this single-center study was conducted at a quaternary care medical center with a robust quality improvement infrastructure. Our interventions took advantage of the existing processes in place that ensure timely discharge of medically ready patients. ${ }^{11}$ Furthermore, microbiology laboratory practices are unique to our institution. These factors limit the generalizability of this work. Second, due to small numbers of eligible infants, analyses were conducted per five patients. Infrequent hospitalizations limited our ability to learn quickly from PDSA cycles. Finally, we did not measure cost savings attributable to shorter hospital stays. However, in addition to financial savings from charges and decreased nonmedical costs such as lost earnings and childcare, ${ }^{17}$ shorter hospitalizations have many additional benefits, such as promoting bonding and breastfeeding and decreasing exposure to nosocomial infections. Shorter hospitalizations, with clearly communicated discharge times, also serve to optimize patient throughput.

\section{CONCLUSION}

Implementation of a clinical practice guideline resulted in reduction of average COT from 38 to 32 hours in febrile infants aged 60 days and younger, with no cases of missed IBI. Engagement of multidisciplinary stakeholders in the generation and structured dissemination of the evidence-based guideline, improved transparency of the microbiological blood and CSF culture process, and standardization of EHR order sets were crucial to the success of this work. Cultures incubated overnight and daily CSF culture-monitoring practices primarily contributed to an average LOS of more than 30 hours.

Future work will include collaboration with emergency physicians to improve evaluation efficiency and decrease LOS in the ED for febrile infants. Additionally, creation of an automated data dashboard of COT and LOS will provide clinicians with real-time feedback on hospitalization practices.

\section{Acknowledgments}

The authors thank Dr Jeffrey Simmons, MD, MSc, as well as the members of the 2019 Fever of Uncertain Source Evidence-Based Guideline Committee. We also thank the James M Anderson Center for Health System Excellence and the Rapid Cycle Improvement Collaborative for their support with guideline development as well as design and execution of our improvement efforts.

Disclosures: The authors have nothing to disclose.

\section{References}

1. Cruz AT, Mahajan P, Bonsu BK, et al. Accuracy of complete blood cell counts to identify febrile infants 60 days or younger with invasive bacterial infections. JAMA Pediatr. 2017;171(11):e172927. https://doi.org/10.1001/ jamapediatrics.2017.2927

2. Kuppermann N, Dayan PS, Levine DA, et al; Febrile Infant Working Group of the Pediatric Emergency Care Applied Research Network (PECARN). A clinical prediction rule to identify febrile infants 60 days and younger at low risk for serious bacterial infections. JAMA Pediatr. 2019;173(4):342-351. https://doi.org/10.1001/jamapediatrics.2018.5501

3. Nigrovic LE, Mahajan PV, Blumberg SM, et al; Febrile Infant Working Group of the Pediatric Emergency Care Applied Research Network (PECARN). The Yale Observation Scale Score and the risk of serious bacterial infections in febrile infants. Pediatrics. 2017;140(1):e20170695. https://doi. org/10.1542/peds.2017-0695

4. De S, Tong A, Isaacs D, Craig JC. Parental perspectives on evaluation and management of fever in young infants: an interview study. Arch Dis Child. 2014;99(8):717-723. https://doi.org/10.1136/archdischild-2013-305736

5. Paxton RD, Byington CL. An examination of the unintended consequences of the rule-out sepsis evaluation: a parental perspective. Clin Pediatr (Phila). 2001;40(2):71-77. https://doi.org/10.1177/000992280104000202

6. FUS Team. Cincinnati Children's Hospital Medical Center. Evidence-based clinical care guideline for fever of uncertain source in infants 60 days of age or less. Guideline 2. 2010:1-4.

7. Aronson PL, Wang ME, Nigrovic LE, et al; Febrile Young Infant Research Collaborative. Time to pathogen detection for non-ill versus ill-appearing infants $\leq 60$ days old with bacteremia and meningitis. Hosp Pediatr. 2018;8(7): 379-384. https://doi.org/10.1542/hpeds.2018-0002

8. Biondi EA, Mischler M, Jerardi KE, et al; Pediatric Research in Inpatient Settings (PRIS) Network. Blood culture time to positivity in febrile infants with bacteremia. JAMA Pediatr. 2014;168(9):844-849. https://doi.org/10.1001/ jamapediatrics.2014.895

9. Lefebvre CE, Renaud C, Chartrand C. Time to positivity of blood cultures in infants 0 to 90 days old presenting to the emergency department: is 36 hours enough? J Pediatric Infect Dis Soc. 2017;6(1):28-32. https://doi. org/10.1093/jpids/piv078

10. Unaka N, Statile A, Bensman, R, et al. Cincinnati Children's Hospital Medical Center. Evidence-based clinical care guideline for evidence-based care guideline for management of infants 0 to 60 days seen in emergency department for fever of unknown source. Guideline 10. 2019;1-42. http://www. cincinnatichildrens.org/service/j/anderson-center/evidence-based-care/ recommendations/default/

11. White CM, Statile AM, White DL, et al. Using quality improvement to optimise paediatric discharge efficiency. BMJ Qual Saf. 2014;23(5):428-436. https://doi.org/10.1136/bmjqs-2013-002556

12. Benneyan JC, Lloyd RC, Plsek PE. Statistical process control as a tool for research and healthcare improvement. Qual Saf Health Care. 2003;12(6): 458-464. https://doi.org/10.1136/qhc.12.6.458

13. Biondi EA, McCulloh R, Staggs VS, et al; American Academy of Pediatrics' Revise Collaborative. Reducing variability in the infant sepsis evaluation (REVISE): a national quality initiative. Pediatrics. 2019;144(3): e20182201. https://doi.org/10.1542/peds.2018-2201

14. McCulloh RJ, Commers T, Williams DD, Michael J, Mann K, Newland JG. Effect of combined clinical practice guideline and electronic order set implementation on febrile infant evaluation and management. Pediatr Emerg Care. 2021;37(1):e25-e31. https://doi.org/10.1097/pec.0000000000002012

15. Foster LZ, Beiner J, Duh-Leong C, et al. Implementation of febrile infant management guidelines reduces hospitalization. Pediatr Qual Saf. 2020;5(1):e252. https://doi.org/10.1097/pq9.0000000000000252

16. Byington $C L$, Reynolds CC, Korgenski K, et al. Costs and infant outcomes after implementation of a care process model for febrile infants. Pediatrics. 2012;130(1):e16-e24. https://doi.org/10.1542/peds.2012-0127

17. Chang LV, Shah AN, Hoefgen ER, et al; H2O Study Group. Lost earnings and nonmedical expenses of pediatric hospitalizations. Pediatrics. 2018;142(3):e20180195. https://doi.org/10.1542/peds.2018-0195 